\title{
Three-candidate competition when candidates have valence: the base case
}

\section{Haldun Evrenk}

Published online: 3 September 2008

(C) Springer-Verlag 2008

\section{Erratum to: Soc Choice Welfare \\ DOI 10.1007/s00355-008-0306-z}

In Evrenk (2008, Proposition 1) "Three-candidate competition when candidates have valence: the base case" (DOI 10.1007/s00355-008-0306-z) the necessary(sufficient) condition for LNE, given as

$$
\frac{-2 f^{\prime}\left(p_{2}^{*}+L^{-1}\left(\delta_{3}\right)\right)}{f\left(p_{2}^{*}+L^{-1}\left(\delta_{3}\right)\right)} \geq(>) \frac{L^{\prime \prime}(0)}{\left[L^{\prime}\left(L^{-1}\left(\delta_{3}\right)\right)\right]^{2}}+\frac{L^{\prime \prime}(0)}{\left[L^{\prime}\left(L^{-1}\left(\delta_{1}\right)\right)\right]^{2}},
$$

is wrong. The correct condition is

$$
\frac{-2 f^{\prime}\left(p_{2}^{*}+L^{-1}\left(\delta_{3}\right)\right)}{f\left(p_{2}^{*}+L^{-1}\left(\delta_{3}\right)\right)} \geq(>) \frac{L^{\prime \prime}(0)}{\left[L^{\prime}\left(L^{-1}\left(\delta_{3}\right)\right)\right]}+\frac{L^{\prime \prime}(0)}{\left[L^{\prime}\left(L^{-1}\left(\delta_{1}\right)\right)\right]},
$$

i.e., the expression $\left[L^{\prime}\left(L^{-1}\left(\delta_{3}\right)\right)\right]$ at the denominator of the terms on the right hand side should not have been raised to the power two. Accordingly, the condition in Remark 1 should read as " . . . becomes $\frac{f^{\prime}\left(-L^{-1}\left(\delta_{1}\right)\right)}{f\left(L^{-1}\left(\delta_{1}\right)\right)}>\frac{L^{\prime \prime}(0)}{\left[L^{\prime}\left(L^{-1}\left(\delta_{1}\right)\right)\right]}$ ".

This error does not affect any of the qualitative or quantitative results in the paper.

It should also be noted that there is a typo in Sect. 3 (Discussion and conclusion). In the example that shows PSNE exists under an asymmetric voter density, the boundaries should be given as $\sqrt{\delta_{1}} \in\left[\frac{44}{100}, \frac{48}{100}\right]$ where in the text it says $\sqrt{\delta_{1}} \in\left[\frac{4}{10}, \frac{48}{100}\right]$.

The online version of the original article can be found under doi:10.1007/s00355-008-0306-z. 